\title{
A STEM-EELS Investigation of La0.7Sro.3CrO3/La0.7Sr0.3MnO3 Multilayer Thin Films
}

Aubrey Penn ${ }^{1}$, Sanaz Koohfar ${ }^{1}$, Divine Kumah ${ }^{1}$ and James LeBeau ${ }^{2}$

${ }^{1}$ North Carolina State University, Raleigh, North Carolina, United States, ${ }^{2}$ Massachusetts Institute of Technology, Cambridge, Massachusetts, United States

La0.7 $\mathrm{Sr}_{0.3} \mathrm{MnO}_{3}$ (LSMO) is a colossal magnetotresistive room temperature ferromagnet with application in magnetic memory and spintronic devices. [1] Growing thin film LSMO epitaxially on structurally similar but chemically different substrates produces new phases of the material and tunes its magnetic properties. Regions of reduced magnetic character, called magnetic dead layers (MDL), form when grown below a critical thickness of about 5 unit cells and near film interfaces. [2] Strain due to lattice mismatch, disruption of bulk oxygen octahedral structure, and interfacial point defects due to the polar discontinuity have each been investigated as possible culprits for the MDL in LSMO. [3,4]. Recently, spacer layers have been introduced between LSMO film and substrate to mitigate the negative interfacial impact on the film's magnetic character. $\mathrm{La}_{0.7} \mathrm{Sr}_{0.3} \mathrm{CrO}_{3}$ (LSCO) has shown to be a good candidate for LSMO heterostructures having a similar lattice constant and octahedral tilting pattern in bulk. Magnetism was reported to be maintained in LSMO-LSCO heterostructures with a LSMO layer thickness of only 2 unit cells due to reduced polar distortions and bulk-like octahedral structure. [5]

In this presentation, we report an investigation of atomic and electronic structure and local chemistry of a [3 LSMO/2 LSCO]x6 multilayered thin film grown by molecular beam epitaxy on $\mathrm{SrTiO}_{3}$ (STO) using X-ray diffraction (XRD) and scanning transmission electron microscopy (STEM) imaging, simulation, and spectroscopy. Figure 1(a-d) shows a high angle annular dark field (HAADF) STEM image of the LSMO-LSCO film with energy dispersive X-ray spectroscopy (EDS) maps of Mn, Cr, and Ti revealing a degree of intermixing between the layers. The in- and out-of-plane (c-axis) lattice parameter determined from atomic column positions in STEM images is shown in Figure 1(e) revealing the film is fully strained with STO in-plane and average out-of-plane parameters of 3.88 $\AA$ and $3.82 \AA$ for LSMO and LSCO layers, respectively. This value for LSMO is 4 pm larger than the XRD-determined value of $3.84 \AA$, while the STEM and XRD determined values for LSCO are in agreement. With electron energy-loss spectroscopy (EELS) it was found that oxygen vacancies preferentially form in LSMO layers, determined from the loss of O K-edge pre-peak intensity at $532 \mathrm{eV}$ (Figure 2). Out-of-plane lattice parameters of 384 and $388 \mathrm{pm}$ correspond to reported values for stoichiometric and oxygen deficient LSMO on STO, respectively. [6]

This discrepancy in measured lattice parameters indicates that oxygen vacancies likely formed during STEM sample preparation performed by mechanical polishing and argon ion milling. LSMO reference films were grown, prepared for STEM, and annealed in oxygen to restore oxygen stoichiometry. Preliminary results for this investigation show a $2 \mathrm{pm}$ decrease in the out-of-plane lattice parameter after annealing. Further simulation and experimentation are required to fully explain the mechanisms underlying the relationship between structure and stoichiometry observed here. Finally, we will highlight how STEM imaging, spectroscopy, and simulation can be used to discover and explain subtle changes in electronic structure and chemistry, and will discuss the importance of combining multiple analysis techniques for accurately determining local and average atomic structure [7]. 
(a)

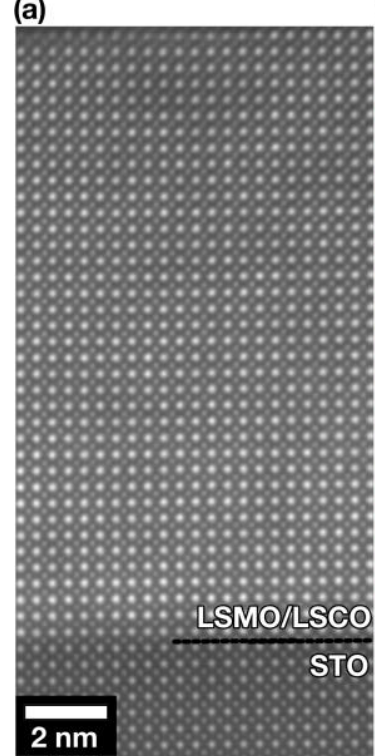

(b)

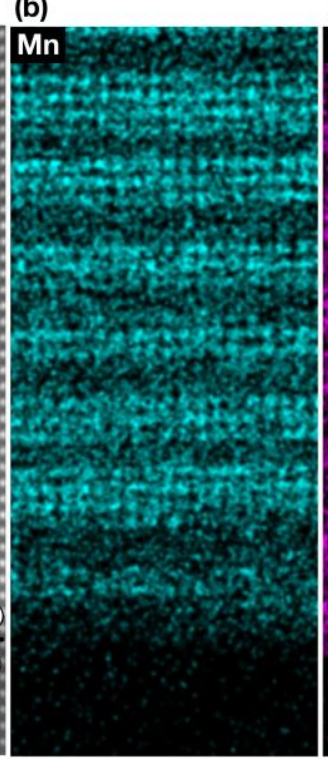

(c)

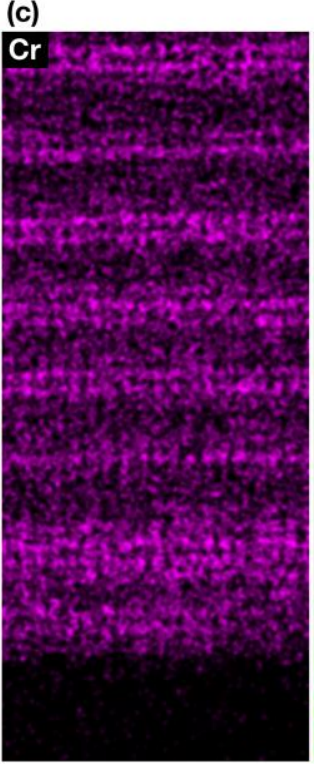

(d)

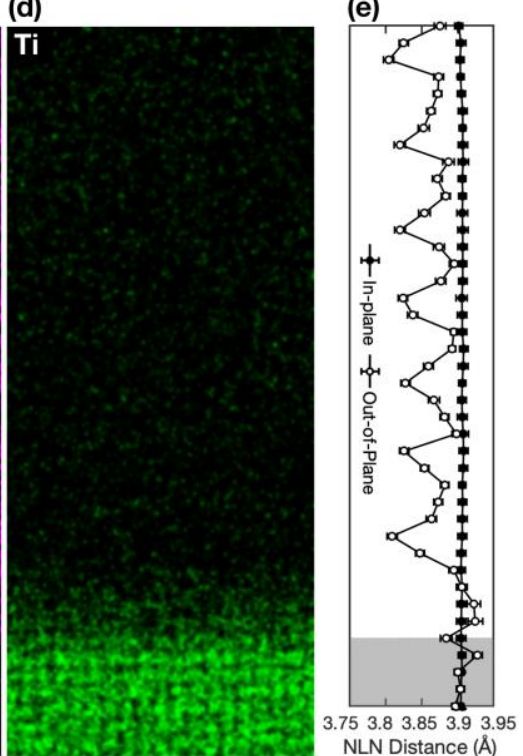

Figure 1. (a) HAADF-STEM image of the [3 LSMO/2 LSCO]x6 multilayered thin film on STO (b) Mn, (c) Cr, and (d) Ti EDS maps (e) In- and out-of-plane lattice parameters determined from the image in (a).

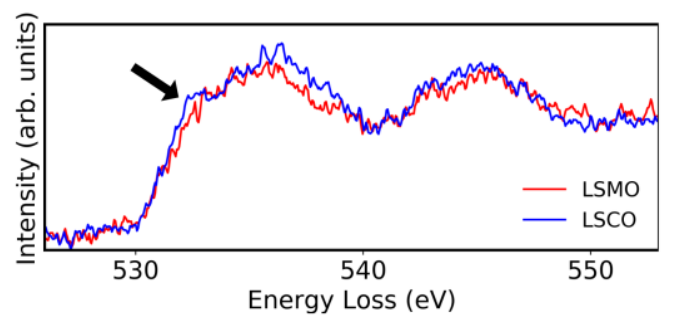

Figure 2. EELS oxygen K-edges revealing an accumulation of oxygen vacancies in LSMO layers.

\section{References}

1. Xiong, Z. H., et al. (2004). Nature, 427(6977), 821-824.

2. Guichard, W., et al. (2002). Journal of Applied Physics, 89(7), 3868-3873.

3. Mundy, J. A., et al. (2014). Nature Communications, 5.

4. Liao, Z., et al. (2016). Nature Materials, 15(4), 425-431.

5. Koohfar, S., et al. (2019). Npj Quantum Materials, 4(1), 25.

6. Orgiani, P., et al. (2012). Applied Physics Letters, 100(4), 042404.

7. This work is supported by the National Science Foundation (DMR-1350273). The authors also acknowledge the Analytical Instrumentation Facility (AIF) at North Carolina State University, which is supported by the State of North Carolina and the National Science Foundation. 